\title{
TITLE: APROTININ OR TRANEXAMIC ACID IN CARDIAC SURGERY: A PROPENSITY ANALYSIS
}

AUTHORS: K Karkouti MD, WS Beattie MD, KM Dattilo MD, SA McCluskey MD, M Ghannam BSc, A Hamdy MD, L Fedorko MD, TM Yau MD

INSTITUTION: Departments of Anesthesia \& Pain Management, and Division of Cardiac Surgery; Toronto General Hospital, University Health Network, University of Toronto, EN 3402, 200 Elizabeth Street, Toronto, Ontario, Canada M5G 2C4

BACKGROUND: Cardiac surgery with cardiopulmonary bypass (CPB) may result in excessive fibrinolysis and platelet dysfunction, resulting in impaired hemostasis and excessive blood loss. Prophylactic use of the antifibrinolytic drugs aprotinin and tranexamic acid is thought to prevent these hemostatic defects. Their relative clinical utility and safety in high-risk cardiac surgery, however, is not known.

METHODS: Following REB approval, propensity score methods were used to match 449 patients who received aprotinin for high-transfusion-risk cardiac surgery to 449 similar patients who received tranexamic, with the patients identified from a pool of 10,870 consecutive patients who underwent cardiac surgery, 586 of whom received aprotinin and the remainder of whom received tranexamic acid. The effects of the two drugs on blood product transfusion and adverse event rates were compared in the matched groups.

RESULTS: The two matched groups were well balanced in terms of all measured perioperative variables. The matched groups had similar rates of blood product transfusion (RBC - Figure platelets and plasma), stroke, myocardial infarction, infection, and mortality. Renal dysfunction (defined as a greater than $50 \%$ increase in creatinine concentration during the first postoperative week to $>100 \mu \mathrm{mol} / \mathrm{L}$ in women and $>110$ in men, or a new requirement for dialysis support) occurred in $24 \%$ of aprotinin patients and $17 \%$ of tranexamic acid patients $(\mathrm{P}=0.01)$. Renal failure requiring dialysis occurred in $6 \%$ and $4 \%$ of the aprotinin and tranexamic acid patients, respectively $(\mathrm{P}=0.1)$.

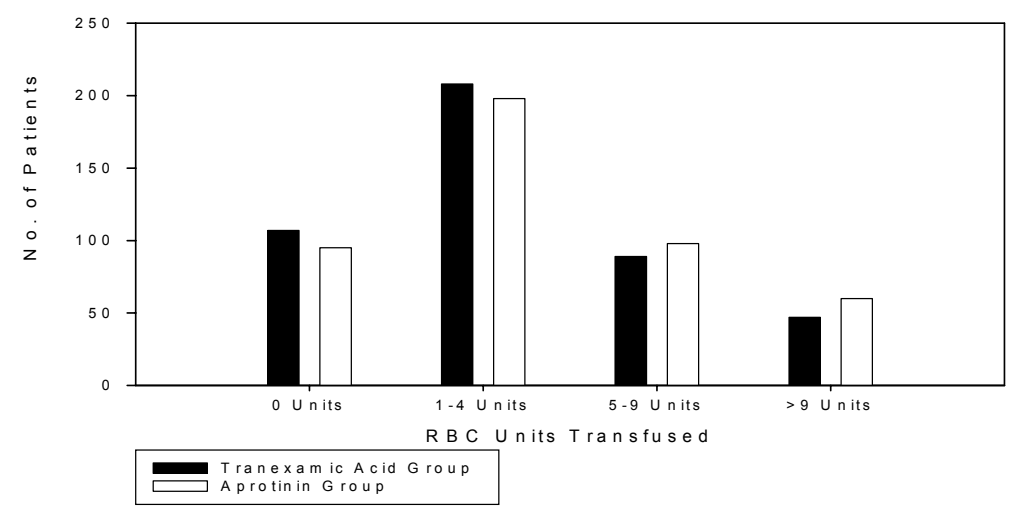

RBC use in matched patients

CONCLUSIONS: In high-risk cardiac surgery, aprotinin offers no clinical advantages over tranexamic acid in terms of its effect on transfusion or morbidity and mortality and is associated with increased risk of renal dysfunction. 